\title{
Microstructural study, tensile properties, and scanning electron microscopy fractography failure analysis of various agricultural residue fibers
}

\begin{abstract}
This paper presents an approach to examine the microstructural properties and mechanical behavior of coconut husks, banana pseudo-stem, pineapple leaf, and sugarcane bagasse fibers by scanning electron microscope and mini-tensile tester, respectively. Single fiber bundles were examined by using scanning electron microscope. Tensile tests were performed at different diameters $(0.15-0.55 \mathrm{~mm})$ and gauge lengths $(10,15,20$, and $30 \mathrm{~mm} / \mathrm{min})$ to assess the effects of diameter and gauge length on tensile properties. It was found that fibers consisted of different types of regularly arranged cells. The tensile strength $(310 \mathrm{MPa})$ and Young's modulus (7.4 GPa) of pineapple leaf fiber bundles showed the highest value compared to the other fibers. The tensile strength and Young's modulus decreased with the increase of diameter and gauge length of fiber bundles. Scanning electron microscopic fractography analysis showed comparatively heterogeneous ruptures associated with more participants of microfibrils for pineapple leaf and banana pseudo-stem fibers compared to coconut husk and sugarcane bagasse fibers. These fractographic observations were discussed in the light of current knowledge of the microstructure of each fiber and the corresponding mechanical properties.
\end{abstract}

Keyword: Agricultural residue; Fiber diameter; Fractography; Gauge length; Mechanical properties; Young's modulus 